\title{
Experimental Study on Prestressed BFRP Reinforced Mental Pipelines
}

\author{
Han Juan ${ }^{1, ~ a, ~ J i a ~ B i n ~}{ }^{2, ~ b}$ \\ ${ }^{1}$ Mianyang Polytechnic, Mianyang, Sichuan, 621010, China \\ ${ }^{2}$ Southwest University of Science and Technology, Mianyang, Sichuan, 621010, China \\ ahanjuan1516@163.com, bjiabin216@126.com
}

Keywords: prestressed BFRP, reinforced, metal pipelines, anchorage

Abstract. Based on the working mechanism of metal pipelines strengthened by basalt fiber reinforced polymers (BFRP), the properties of the new stretching and anchoring integral anchorage for prestressed BFRP are studied. The results show that BFRP could be prestessed by rotating anchor rod, and that bolt could anchor BFRP effectively. And the strain distribution law of prestressed BFRP is analyzed. It provides the test basis for the design and construction of prestressed BFRP reinforced metal pipelines.

\section{Introduction}

Metal pipelines have been widely used in the fields of municipal engineering, petrochemical industry and water conservancy ${ }^{[1,2]}$. The metal pipelines in service are inevitably damaged, and it is necessary to solve the technical problems in the engineering field to seek economical and efficient repairing technology of metal pipelines. BFRP reinforced metal pipelines has the advantages of simple transmission, corrosion resistance and easy construction.

At present, there are few researches on prestressed BFRP reinforced mental pipelines at home and abroad, the key factor is the lack of effective method of applying circumferential prestress, while the method of prestressed BFRP reinforced mental pipelines is safer than wrapping with BFRP ${ }^{[3,4]}$. Therefore, the development of an effective prestressed device becomes the precondition of prestressed BFRP reinforced mental pipelines.

Based on the mechanics principle and method of BFRP reinforced mental pipelines, combining with the advantages of the existing prestressed device, the research group developed a set of stretching and anchoring BFRP with circumferential prestress. The paper introduces its working mechanism and analyzes the strain distribution of prestressed BFRP reinforced mental pipelines.

\section{Design of stretching and anchoring integral anchorage}

\section{Device design and production}

Based on the mechanical principle of BFRP reinforced mental pipelines with internal pressure, and considering the stress characteristics of mental pipelines, a stretching and anchoring integral anchorage applying prestress is put forward under the precondition of guaranteeing long-term stability of prestress. The integral anchorage is composed of bottom plate, anchor plate, anchor rod and bolt.

The test apparatus uses Q235 steel, the length of the anchor rod is $260 \mathrm{~mm}$, and the diameter is $30 \mathrm{~mm}$; the thickness of the anchor plate is $20 \mathrm{~mm}$, the width is $100 \mathrm{~mm}$, and the height $50 \mathrm{~mm}$; the diameter of A-class bolts is $12 \mathrm{~mm}$, and the length is $50 \mathrm{~mm}$; the thickness of the bottom plate is $200 \mathrm{~mm}$; the E43 electrode is selected and welded manually, the weld form is fillet weld, the leg length is $10 \mathrm{~mm}$, and the two ends are not used run-on plate, each anchor plate is welded on both sides.

\section{The working principle of the anchorage}

The principle of stretching and anchoring BFRP with anchorage is that BFRP can be tensioned beforehand to produce prestress by rotating anchor rod, and when the prestress reaches predetermined value, the anchor rod is fixed by using bolts and prestressed BFRP is locked avoiding the loss of prestress due to the shrinkage of BFRP.

When the anchorage is applied to BFRP reinforced mental pipelines, BFRP is subjected to tension and the interface of mental pipelines is under positive pressure. The anchor base is under pressure from 
the upper anchor rod, and the interface is under positive pressure on the contact interface of the anchorage and mental pipeline to form a closed ring-shaped confinement effect. The stretching and anchoring device for prestressing is simple in structure, concise in transmission force and reliable in anchoring prestressed BFRP, and it is simple and easy to operate.



Fig. 1 The working principle of the anchorage

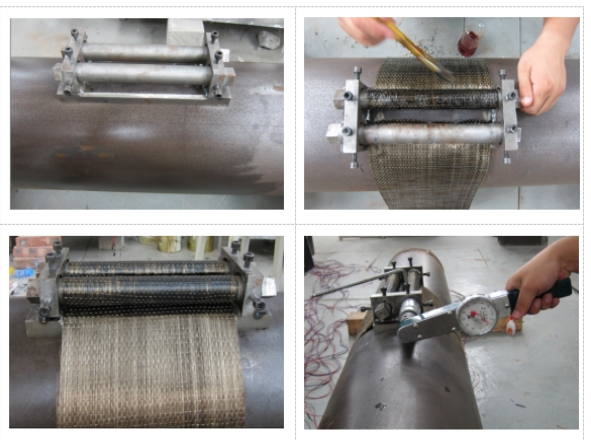

Fig. 2 The construction operating procedure

\section{The construction technology of the anchorage applying prestress}

The construction operating procedure of the anchorage applying prestress are as follow, as shown in Fig. 2 .

(1) Polish and clean the surface of mental pipeline to be strengthened with alcohol, and fix the anchorage in a reinforced area.

(2) Cut the BFRP sheet with corresponding length.

(3) Configure glue separately with epoxy resin $\mathrm{AB}$ group.

(4) Wrap BFRP around the mental pipeline to be reinforced, and paste the ends of BFRP with the glue to the anchor rod.

(5) Fix one end of the anchor rod and rotate the other end when the glue is solidified.

(6) When prestress reaches the preset value, promptly tighten the bolt to lock the anchor rod.

\section{Experimental study on prestressed BFRP reinforced mental pipelines with anchorage}

\section{Test Overview}

Test materials and equipment

The specimen is made of Q235B low carbon steel pipes with a dimension of $273 \mathrm{~mm} \times 1.92 \mathrm{~mm} \times 1500$ $\mathrm{mm}$, the elastic modulus is $200 \mathrm{GPa}$, the yield strength is $240 \mathrm{MPa}$, and the ultimate strength is 445 $\mathrm{MPa}$. The tensile strength of BFRP is $2002 \mathrm{MPa}$, and the elastic modulus is $105 \mathrm{GPa}$. The glue uses $\mathrm{CH}-1 \mathrm{~A}$ resin-impregnated with a shear strength of $24.6 \mathrm{MPa}$.

The test equipment adopts the static strain tester of DH3818-3 produced by Donghua Corporation and the ACD and TG300 torque wrench produced by Zhejiang Shengzhou Moment Tool Manufacturing Co., LTD.

Loading method

According to the operating procedure of the anchorage applying prestress, and the arrangement of strain gauge is shown in Fig. 3. The torque is applied to the anchor rod by using graded step-by-step loading method with the torque wrench.

\section{Test results and analysis}

The validity of stretching and anchoring integral anchorage

When the torque is applied to $160 \mathrm{~N} \cdot \mathrm{m}$, the change of BFRP strain in $2 \mathrm{~h}$ is shown in Fig. 4. BFRP is tensile strain by stretching, and the loss of strain after anchorage locked is reduced, but the strain attains balance after $2 \mathrm{~h}$, and remains in the initial value of $95.54 \%$. It indicates that the stretching and anchoring integral anchorage is effective and feasible. 


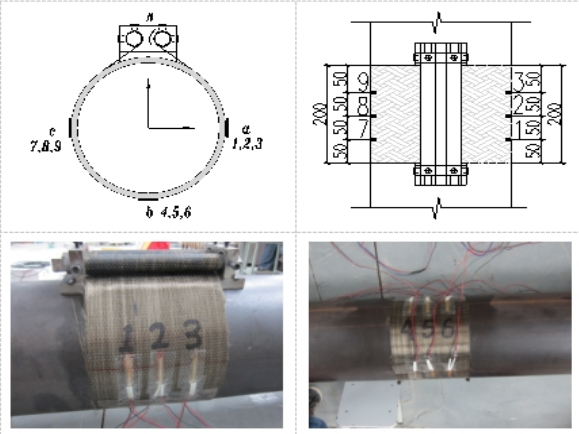

Fig. 3 Arrangement of measuring points

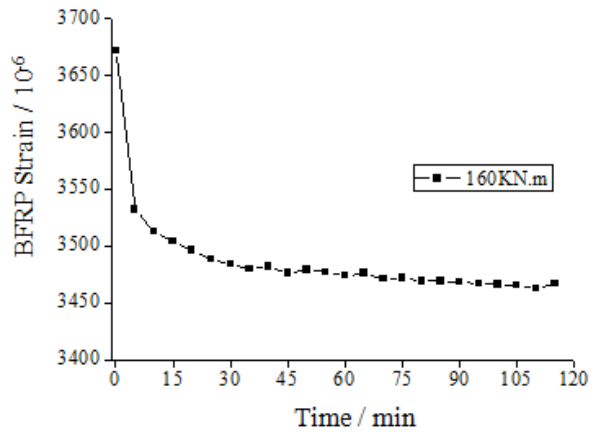

Fig. 4 The change curve of strain and time

The strain distribution of prestressed BFRP

According to the prestressing steps, the loading ranges from 0 to $120 \mathrm{~N} \cdot \mathrm{m}$, and the test is carried out by using the torque wrench, and the strain distribution of prestressed BFRP has the following rules.

(1) The BFRP strain value is not evenly distributed on the axial section.

Under the same torque, the BFRP strain values of different axial positions on the same circumference are different, as shown in Table 1. The reason is that BFRP sheet is different from the general homogeneous material, which is made of many root filaments, and it is difficult to achieve the same length in weaving process. The filaments of BFRP without brushing glue are independent of each other, and they cannot be coordinated as a whole. Therefore, it is very difficult for the BFRP to be uniformly prestressed when the tension of the anchorage is not equal to that of each fiber.

Table 1 The strain measured results of measuring points

\begin{tabular}{cccccccccc}
\hline $\begin{array}{c}\text { Torque } \\
\text { /N.m }\end{array}$ & $\mathbf{1}$ & $\mathbf{2}$ & $\mathbf{3}$ & $\mathbf{4}$ & $\mathbf{5}$ & $\mathbf{6}$ & $\mathbf{7}$ & $\mathbf{8}$ & $\mathbf{9}$ \\
\hline 0 & 6 & 9 & 15 & 2 & 19 & 0 & 0 & 9 & 0 \\
5 & 324 & 264 & 304 & 158 & 144 & 121 & 164 & 160 & 166 \\
10 & 517 & 422 & 434 & 235 & 205 & 176 & 250 & 243 & 292 \\
15 & 693 & 576 & 584 & 308 & 318 & 257 & 366 & 310 & 333 \\
20 & 884 & 767 & 828 & 340 & 426 & 372 & 562 & 436 & 402 \\
25 & 1021 & 884 & 933 & 411 & 496 & 424 & 660 & 522 & 474 \\
30 & 1115 & 982 & 1047 & 443 & 548 & 471 & 712 & 567 & 531 \\
35 & 1182 & 1060 & 1120 & 483 & 609 & 533 & 756 & 597 & 569 \\
40 & 1171 & 1028 & 1103 & 446 & 592 & 541 & 766 & 551 & 466 \\
45 & 1384 & 1178 & 1298 & 534 & 692 & 613 & 918 & 774 & 687 \\
50 & 1391 & 1292 & 1401 & 566 & 784 & 682 & 943 & 850 & 758 \\
55 & 1416 & 1279 & 1395 & 571 & 801 & 720 & 984 & 878 & 789 \\
60 & 1512 & 1393 & 1491 & 586 & 839 & 734 & 1035 & 916 & 820 \\
70 & 1532 & 1231 & 1229 & 725 & 1059 & 1067 & 1377 & 1257 & 1081 \\
80 & 2048 & 1805 & 1789 & 978 & 1307 & 1239 & 1704 & 1519 & 1347 \\
90 & 2159 & 1929 & 1928 & 973 & 1317 & 1212 & 1708 & 1532 & 1379 \\
100 & 2541 & 2260 & 2183 & 1218 & 1482 & 1294 & 1883 & 1677 & 1566 \\
110 & 2655 & 2278 & 2153 & 1249 & 1561 & 1371 & 1986 & 1766 & 1682 \\
120 & 2732 & 2459 & 2385 & 1449 & 1728 & 1547 & 2181 & 1921 & 1813 \\
\hline
\end{tabular}


(2) The strain value is not evenly distributed in the circular direction.

In order to reduce the influence of strain on axial cross section distribution, it is assumed that the average axial cross-sectional strain is the strain of the BFRP section. Under the same torque action, the strain value of BFRP in the distance from the anchorage is smaller, as shown in Fig. 5. This is because there is the friction between BFRP and the contact surface of the hardened specimen, and with the gradual tension of BFRP, the friction force is increasing.

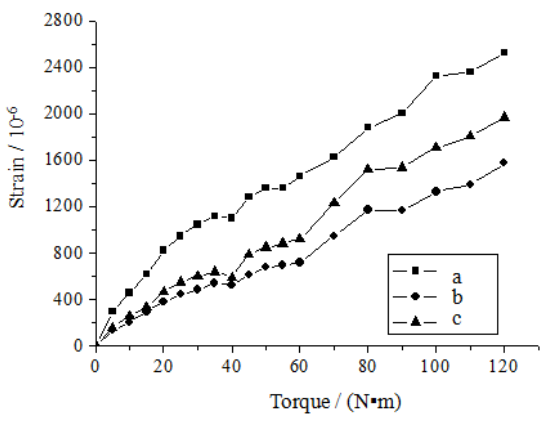

Fig. 5 The torque and strain curve of different measuring position

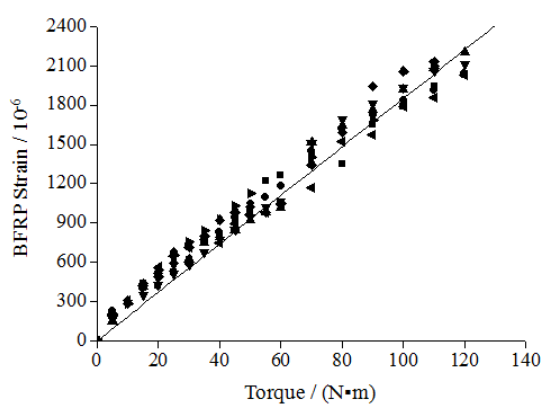

Fig. 6 The curve of torque and the average BFRP strain

(3) The average strain is linearly $r$ elated to the torque value.

The average strain of BFRP increases with the increased torque, and the distribution between them is close to the obvious linear relationship, as shown in Fig. 6.

\section{Conclusion}

Based on the working mechanism of BFRP reinforced metal pipelines, BFRP applying prestress is proposed by using stretching and anchoring integral anchorage. The paper expounds the working principle and the construction technology applying prestress of the anchorage, and establishes the BFRP strain distribution law after applying prestress and analyzes the causes. It provides an important reference for the further study on the technical method of prestressed BFRP reinforced metal pipelines.

\section{References}

[1] Chow Dhury S G, Mukhopadhyay, Bhattacharya D K. Failure Analysis of a Weld Repaired Steam Turbine Casing [J]. Engineering Failure Analysis, 1998, 5(3):205-218.

[2] Clubley S K, Winter S N. On the Fatigue and Fracture of Site Splice Welds at the River Mardle Viaduel Engineering [J]. Failure Analysis, 2003, 10(5): 593-604.

[3] Yang Yongxin, Peng Fuming, Yue Qingrui. Stress Analysis of Fiber Reinforced Composite Reinforced Metal Pipelines [J]. Port Engineering Technology, 2005(4): 17-19.

[4] Jia Bin, Zhang Zhiwei, Chen Xiaoqiang. Design and Experimental Study on Mental Pipeline Strengthened by Fiber Reinforced Polymers [J]. Industrial Construction, 2013, 43(7): 57- 60. 